\title{
DIAGNÓSTICO DE LA FASE DE PRE INVERSIÓN EN HOLGUÍN. UN ENFOQUE DE PROCESOS
}

\section{DIAGNOSIS OF PRE INVESTMENT PHASE IN HOLGUIN. A PROCESS APPROACH}

\author{
Luis Alexis Aguilera García \\ Universidad de Holguín Facultad de Ciencias \\ Económicas y Administración \\ Universidad de Holguín, Holguín, Cuba \\ lagarcia@uho.edu.cu \\ Maira Rosario Moreno Pino \\ Facultad de Ciencias Económicas y Administración \\ Universidad de Holguín, Holguín, Cuba \\ mpino@uho.edu.cu
}

\author{
Yosvani Orlando Lao León \\ Facultad de Ciencias Económicas y Administración \\ Universidad de Holguín, Holguín- Cuba, \\ ylaol@uho.edu.cu \\ Yumelys Lores Rodríguez \\ Servicios Especializados de Seguridad (SEISA) \\ Ciudad de Holguín, Provincia Holguín, Cuba \\ yumelis@seisa.cu
}

Fecha de recepción: 20/12/2020 - Fecha de revisión: 19/01/2021 - Fecha de aprobación: 25/01/2021

DOI: https://doi.org/10.36995/j.visiondefuturo.2021.25.02R.003.es

\section{RESUMEN}

El objetivo de este artículo es realizar un estudio de diagnóstico con un enfoque de procesos de la fase de pre inversión en la provincia de Holguín, Cuba, comparándolo con otros subprocesos de pre inversión en América Latina. Para esto se emplearon métodos y técnicas como el coeficiente de concordancia de Kendall, el análisis DAFO y la Casa de la Calidad. Como principales resultados se obtuvieron el estado actual de la calidad del subproceso pre inversión en el contexto holguinero así como y el perfil competitivo con respecto a otros subprocesos de pre inversión en la región. Se concluye que el subproceso de pre inversión en Holguín posee como principal deficiencia la baja calidad de los estudios de pre inversión, aspecto que a su vez constituye la característica más representativa en el diseño de este subproceso.

PALABRAS CLAVE: Proceso inversionista; Fase de pre inversion; Diseño de procesos.

\section{ABSTRACT}

The objective of this article is to perform a diagnostic study with a focus on preinvestment phase processes in the province of Holguín, Cuba, comparing it with other preinvestment sub-processes in Latin America. For this, methods and techniques such as Kendall's coefficient of concordance, the SWOT analysis and the House of Quality were used. The main results were the current status of the quality of the pre-investment sub-process in the Holguin context, as well as the competitive profile with respect to other pre-investment subprocesses in the region. It is concluded that the pre-investment sub-process in Holguín has as

\footnotetext{
"Visión de Futuro" Año 18, Volumen No 25 N², Julio - Diciembre 2021 - Pág 81 - 99 URL de la Revista: http://visiondefuturo.fce.unam.edu.ar/index.php/visiondefuturo/index URL del Documento: https://visiondefuturo.fce.unam.edu.ar/index.php/visiondefuturo/issue/view/21 ISSN 1668 - 8708 - Versión en Línea E-mail: revistacientifica@fce.unam.edu.ar
} Creative Commons Atribuci 
its main deficiency the low quality of the pre-investment studies, an aspect that in turn constitutes the most representative characteristic in the design of this sub-process.

KEY WORDS: Diagnosis; Investment process; Pre investment subprocess; Process approach.

\section{INTRODUCCIÓN}

El objetivo de la presente investigación consiste en la realización de un diagnóstico de la fase de pre inversión con un enfoque de procesos en la provincia de Holguín, Cuba. Esta fase constituye el inicio del proceso inversionista y a su vez un subproceso para la adquisición de certidumbre a través de evaluaciones relacionadas con la viabilidad de la inversión en cuestión, que derivan en la decisión crítica de invertir o no. Cualquier imprecisión cometida en esta fase es arrastrada hacia las demás, aspecto que deriva en una distorsión, en ocasiones irremediable, de la idea inicial de la inversión y de los resultados esperados (López et al., 2012; Sáenz, 2013; Sánchez y Ledesma, 2015a; Duffus et al., 2018; Ochoa, 2019; Rodríguez, 2019).

La experiencia práctica a escala global refiere que no basta disponer de recursos suficientes para el alcance de los objetivos de un proyecto de inversión, sino que resulta también indispensable la calidad de los análisis y estudios realizados en la fase de pre inversión, y la calidad en la ejecución de estos en las fases posteriores; por cuanto, esta fase constituye la base estructural del proceso y un punto de inflexión para el diagnóstico del proceso en general (Secretaría de Planificación y Programación de la Presidencia en Guatemala, 2002; Thompson, 2009; Apaza, 2015; Cámara Colombiana de la Infraestructura, 2016).

En la provincia de Holguín se desarrollan actualmente importantes procesos inversionistas en sectores estratégicos como el turismo, la salud y la agricultura. El dinamismo y las interconexiones de estos proyectos de inversión demandan una estructura más eficiente y competitiva de la fase de pre inversión como subproceso elemental, basada en un diagnóstico oportuno de la misma. A pesar los elementos antes expuestos, no constituye una práctica habitual en las organizaciones holguineras el diagnóstico de la fase de pre inversión como subproceso clave del proceso inversionista en general, ni como una herramienta para la identificación de errores y desviaciones así como, oportunidades de mejora, aspecto que ha comprometido en ocasiones, importantes y escasos recursos en procesos inversionistas fallidos. Esta situación se evidencia en la coexistencia de deficiencias en el desarrollo de esta

\footnotetext{
"Visión de Futuro" Año 18, Volumen No 25 N², Julio - Diciembre 2021 - Pág 81 - 99

URL de la Revista: http://visiondefuturo.fce.unam.edu.ar/index.php/visiondefuturo/index

URL del Documento: https://visiondefuturo.fce.unam.edu.ar/index.php/visiondefuturo/issue/view/21

ISSN 1668 - 8708 - Versión en Línea

E-mail: revistacientifica@fce.unam.edu.ar
} 
fase. Estas pueden agruparse en:

\section{Calidad técnica de los estudios de pre inversión}

- Estudios de factibilidad con bajo rigor y nivel de profundidad (Valarezo et al., 2016);

- No se poseen estándares de calidad comunes para la fase de pre inversión que funcionen como requisitos indispensables para el desarrollo exitoso de esta fase (Ochoa, 2019; Rodríguez, 2019; Aguilera et al., 2020);

- Las brechas existentes en la evaluación de los estudios realizados en la fase de pre inversión provoca la aceptación de estudios de mercado superficiales sin una cuantificación y argumentación correcta de la(s) demanda(s) identificada(s) (Duffus et al., 2018);

- Procedimientos inadecuados para el cálculo de la depreciación que conlleva errores en la estimación de impuestos, reposiciones y valores residuales, traduciéndose en resultados con un margen de error mayor de lo planificado (Sáenz, 2013);

\section{Fiabilidad de los resultados}

- Dispersión de la información necesaria para la realización de los análisis y pronósticos, aspecto que deriva en una mayor imprecisión en la evaluación final contenida en estos estudios (Sánchez et al., 2015a);

- La no realización de evaluaciones integrales de los estudios y análisis realizados en la fase de pre inversión, dificulta el análisis de la fiabilidad de la información resultante (Sánchez y Ledesma, 2018);

- La no existencia de coeficientes ramales dificulta la correcta aplicación de la tasa de descuento, aspecto que distorsiona la viabilidad de la inversión, y en ocasiones, su resultado final (Martín y Leyva, 2017).

\section{Desfasajes en los plazos}

- El débil cumplimiento de los cronogramas provoca importantes desfasajes en la fase de pre inversión que atentan contra la correcta ejecución del presupuesto y la calidad de la fase en general (Sánchez y Ledesma, 2015b);

- Los retrasos en la entrega de la documentación técnica para la elaboración de los estudios de pre inversión, influye en la dilatación de la fase de pre inversión;

\footnotetext{
"Visión de Futuro" Año 18, Volumen N$^{0} 25$ No 2, Julio - Diciembre 2021 - Pág 81 - 99 URL de la Revista: http://visiondefuturo.fce.unam.edu.ar/index.php/visiondefuturo/index URL del Documento: https://visiondefuturo.fce.unam.edu.ar/index.php/visiondefuturo/issue/view/21 ISSN 1668 - 8708 - Versión en Línea 
- La gerencia del proceso inversionista, carece en ocasiones de un enfoque integral, que involucre a todas las partes interesadas en la sincronización de las actividades y su cumplimiento (Sardiñas, 2016).

Por tanto, resulta necesario el enfoque de procesos en el diagnóstico de la fase de pre inversión para la identificación de oportunidades de mejora y erradicación a tiempo, de desviaciones en los resultados esperados, en el contexto holguinero. Estos elementos contribuyen al logro de un subproceso de mayor calidad.

\section{DESARROLLO}

\section{1.- Antecedentes}

Las decisiones relacionadas con los procesos inversionistas comprenden análisis y proyecciones del uso racional, presente y futuro de recursos materiales, humanos y financieros, para la obtención de utilidades, desde una perspectiva social, económica y ambiental sostenible (Peña et al., 2015). Cada fase del proceso constituye un subproceso del mismo y tributa al cumplimiento de los objetivos que motivaron la inversión (Paño, 2016). Si bien todas las fases son importantes, la fase de pre inversión, como eslabón inicial del proceso y como fuente de información esencial para las siguientes fases, constituye un punto vertebral en el éxito del proyecto de inversión (Thompson, 2009; Sapag, 2011; Vera, 2015; Cabrera y Paredes, 2016; Villafuerte, 2016; Wang et al., 2018; Aguilera et al., 2020).

Cualquier imprecisión cometida en esta fase, posee generalmente un carácter irreversible, reflejándose en el resto de las fases (subprocesos) cuando sus efectos son prácticamente inevitables (López et al., 2012; Sáenz, 2013; Sánchez et al., 2015a; Duffus et al., 2018; Ochoa, 2019; Rodríguez, 2019), por tanto, su análisis desde el enfoque de procesos resulta pertinente. En la literatura consultada diversos autores refieren que resulta oportuno el empleo de herramientas convencionales de la gestión empresarial como el análisis DAFO (Olivera y Matamoros, 2011; Foschiatti y Alberto, 2012) y la Casa de la Calidad o QFD (Quality Function Deployment) (Shen et al., 2001; Reich y Levy, 2004; Kim et al., 2007) para el análisis y diseño de procesos respectivamente (Sarmiento et al., 2004; Kim et al., 2007; Elizondo, 2019). No obstante, en las fuentes bibliográficas a las que se tuvo acceso, no se constata el uso del enfoque de procesos en el diagnóstico del proceso inversionista, y específicamente de la fase de pre inversión, sino que tienden a analizarse meramente desde indicadores económicos.

\footnotetext{
"Visión de Futuro" Año 18, Volumen No 25 N 2, Julio - Diciembre 2021 - Pág 81 - 99 URL de la Revista: http://visiondefuturo.fce.unam.edu.ar/index.php/visiondefuturo/index URL del Documento: https://visiondefuturo.fce.unam.edu.ar/index.php/visiondefuturo/issue/view/21 ISSN 1668 - 8708 - Versión en Línea E-mail: revistacientifica@fce.unam.edu.ar
} 


\section{2.- Materiales y métodos}

En el desarrollo de la investigación se empleó el método de coeficiente de concordancia de Kendall con escala de ponderación descendente para evaluar el consenso de los expertos y la resolución de contradicciones, que inciden en el problema planteado y buscar soluciones teórico-prácticas que contribuyan a la solución de este problema. Se empleó la matriz DAFO para el diagnóstico de la fase de pre inversión en el contexto holguinero y la Casa de la Calidad (QFD) para la identificación de oportunidades de mejora que contribuyan a la calidad y asertividad del proceso. Otros métodos teóricos como: análisis y síntesis, inductivo-deductivo y el sistémico estructural se emplearon para el análisis de las referencias documentales en la literatura especializada asociada al proceso inversionista y la fase de pre inversión.

\section{3.- Selección del grupo de expertos}

Para el diagnóstico de la fase de pre inversión en la provincia de Holguín se seleccionaron nueve expertos, tres doctores en ciencias técnicas, cuatro doctores en ciencias económicas y dos inversionistas del sector empresarial en funciones. Se tuvo en cuenta la trayectoria laboral y la experiencia de cada experto en su rama, así como su relación directa e indirecta con el proceso inversionista. Los expertos se agrupan en tres centros laborales: siete procedentes de la Universidad de Holguín, uno del Gobierno Provincial y uno de la Asociación Nacional de Economistas de Cuba.

\section{4.- Recogida de datos}

Para identificar las principales fortalezas, debilidades, amenazas y oportunidades de la fase de pre inversión en Holguín se consultó a los expertos seleccionados a través de la aplicación de un instrumento (figura 1). Este fue validado a través del coeficiente alfa de Cronbach obteniéndose un valor de $\alpha=0.76$ por tanto, la encuesta es fiable. Se empleó el método del coeficiente de concordancia de Kendall para evaluar el nivel de consenso entre estos.

\footnotetext{
"Visión de Futuro" Año 18, Volumen No 25 N² 2, Julio - Diciembre 2021 - Pág 81 - 99

URL de la Revista: http://visiondefuturo.fce.unam.edu.ar/index.php/visiondefuturo/index

URL del Documento: https://visiondefuturo.fce.unam.edu.ar/index.php/visiondefuturo/issue/view/21

ISSN 1668 - 8708 - Versión en Línea

E-mail: revistacientifica@fce.unam.edu.ar
} 


\section{CUESTIONARIO}

Estimado colaborador tenemos a bien recoger sus consideraciones respecto al proceso inversionista con el objetivo de identificar y ponderar las principales fortalezas $(F)$-debilidades $(D)$ y oportunidades $(O)$-amenazas $(A)$ más representativas que presenta la fase de pre inversión en la provincia de Holguín.

Agradecemos su colaboración en la respuesta de este cuestionario y el esfuerzo en reportar los datos con alta confiabilidad. Los resultados obtenidos de su aplicación serán manejados con total confidencialidad ya que el estudio es estrictamente académico.

Instrucciones: lea cuidadosamente todo el documento y relacione las $F, D, O$ y $A$ y pondere a partir de su consideración de en qué medida impactan en el desarrollo de la fase de pre inversión en la provincia Holguín (1. Muy débil 2. Débil 3. Medio 4. Fuerte 5. Muy fuerte)

Fortalecimiento de la relación Universidad-Empresa $(F)$

Incremento del volumen de procesos inversionistas en la provincia de Holguín en los sectores estratégicos $(F)$

Mayor autonomía otorgada a los gobiernos provinciales por el Estado con respecto a la gestión de los procesos inversionistas desde la fase inicial (pre inversión) (F)

Desarrollo de proyectos intersectoriales como Trasvase Este-Oeste y Polo turístico Ramón de Antilla ( $F$ )

Potenciamiento de servicios de consultoría en la realización de estudios de pre inversión $(F)$

Incumplimiento de los cronogramas y plazos establecidos (D)

Exceso de trámites burocráticos para concretar oportunidades de inversión (D)

Brechas en el proceso de capacitación en el personal que participa en la realización de los estudios de pre inversión (D)

Deficiencias en la gestión de la información inherente a la fase de pre inversión (D)

Dispersión, poca profundidad y bajo rigor en los estudios de pre inversión (D)

Cambio de la matriz energética del país aspecto que influye en el desarrollo de proyectos con energías renovables en la provincia de Holguín (O)

Orientación de la política del Estado hacia el desarrollo exitoso del proceso inversionista y dentro de este la fase de pre inversión (O)

La globalización de los servicios profesionales permite el intercambio fructífero de experiencias en cuanto al proceso inversionista y la ejecución de la fase de pre inversión (O)

La potencialidad de la inversión extranjera en el territorio presupone una mayor preparación de los inversionistas en cuanto a los estudios ingenieros así como, de pre y factibilidad técnico-económica $(O)$

\begin{tabular}{|c|c|c|c|}
\hline & 2 & 3 & \\
\hline & 2 & 3 & 4 \\
\hline & 2 & 3 & 4 \\
\hline & 2 & 3 & 4 \\
\hline$T$ & 2 & 3 & 4 \\
\hline 1 & 2 & 3 & 4 \\
\hline & 2 & 3 & 4 \\
\hline 1 & 2 & 3 & 4 \\
\hline 1 & 2 & 3 & 4 \\
\hline 1 & 2 & 3 & 4 \\
\hline 1 & 2 & 3 & 4 \\
\hline 1 & 2 & 3 & 4 \\
\hline 1 & 2 & 3 & 4 \\
\hline 1 & 2 & 3 & 4 \\
\hline 1 & 2 & 3 & 4 \\
\hline 1 & 2 & 3 & 4 \\
\hline 1 & 2 & 3 & 4 \\
\hline 1 & 2 & 3 & 4 \\
\hline 1 & 2 & 3 & 4 \\
\hline 1 & 2 & 3 & 4 \\
\hline
\end{tabular}

Realización de investigaciones científicas y empíricas sobre el desempeño de la fase de pre inversión $(\mathrm{O})$

Recrudecimiento del bloqueo económico de Estados Unidos contra Cuba (A)

Crisis económico-sanitaria global $(\mathrm{A})$

Inestabilidad en la cadena de suministros $(A)$

Dualidad monetaria (A)

Limitaciones en la capacidad constructiva del país $(A)$

\footnotetext{
"Visión de Futuro" Año 18, Volumen No 25 No 2, Julio - Diciembre 2021 - Pág 81 - 99

URL de la Revista: http://visiondefuturo.fce.unam.edu.ar/index.php/visiondefuturo/index

URL del Documento: https://visiondefuturo.fce.unam.edu.ar/index.php/visiondefuturo/issue/view/21

ISSN 1668 - 8708 - Versión en Línea

E-mail: revistacientifica@fce.unam.edu.ar
} 
Figura $N^{\circ}$ 1: Encuesta para identificar los factores que afectan la ejecución de la fase de pre inversión en Holguín

Fuente: Elaboración propia

Para esto fue empleada una escala de ponderación (1-10) descendiente, se obtuvo un coeficiente de concordancia de Kendall de 0.97 por tanto existió consenso en el criterio del grupo de expertos, este fue validado a partir de la prueba no paramétrica correspondiente (chi-cuadrado), donde el estadígrafo calculado (20.64) resultó mayor que el tabulado (7.86). Los expertos coinciden que las fortalezas-debilidades y oportunidades-amenazas así como su ponderación son las relacionadas en la tabla 1 y 2.

Tabla No1: Ponderación de factores internos

\section{Fortalezas} Sg $P$

Fortalecimiento de la relación Universidad-Empresa F1 3

Incremento del volumen de procesos inversionistas en la provincia de Holguín en los sectores estratégicos

Mayor autonomía otorgada a los gobiernos provinciales por el Estado con respecto a la gestión de los procesos inversionistas desde la fase inicial (pre inversión)

Desarrollo de proyectos intersectoriales como Trasvase Este-Oeste y Polo turístico Ramón de Antilla

Potenciamiento de servicios de consultoría en la realización de estudios de pre inversión

\section{Debilidades}

Incumplimiento de los cronogramas y plazos establecidos

Exceso de trámites burocráticos para concretar oportunidades de inversión

Brechas en el proceso de capacitación en el personal que participa en la realización de los estudios de pre inversión

Deficiencias en la gestión de la información inherente a la fase de pre inversión

Dispersión, poca profundidad y bajo rigor en los estudios de pre inversión

Tabla N²: Ponderación de factores externos

\begin{tabular}{|ccc|}
\hline Oportunidades & Sg & $\mathbf{P}$ \\
\hline $\begin{array}{c}\text { Cambio de la matriz energética del país aspecto que influye en el desarrollo de proyectos } \\
\text { con energías renovables en la provincia de Holguín }\end{array}$ & $\mathrm{O} 1$ & 3 \\
$\begin{array}{c}\text { Orientación de la política del Estado hacia el desarrollo exitoso del proceso inversionista y y } \\
\text { dentro de este la fase de pre inversión }\end{array}$ & $\mathrm{O} 2$ & 4 \\
La globalización de los servicios profesionales permite el intercambio fructífero de & $\mathrm{O} 3$ & 3 \\
$\begin{array}{c}\text { experiencias en cuanto al proceso ínversionista y la ejecución de la fase de pre inversión } \\
\text { La potencialidad de la inversión extranjera en el territorio presupone una mayor }\end{array}$ & $\mathrm{O} 4$ & 4 \\
preparación de los inversionistas en cuanto a los estudios ingenieros así como, de pre y & & \\
factibilidad técnico-económica & & \\
Realización de investigaciones científicas y empíricas sobre el desempeño de la fase de & $\mathrm{O}$ & 4 \\
pre inversión & & \\
Amenazas & $\mathrm{Sg}$ & $\mathbf{P}$ \\
A1 & 1 \\
Recrudecimiento del bloqueo económico de Estados Unidos contra Cuba & 1 \\
Crisis económico-sanitaria global & $\mathrm{A} 3$ & 2 \\
Inestabilidad en la cadena de suministros & $\mathrm{A} 4$ & 1 \\
Dualidad monetaria & $\mathrm{A} 5$ & 2
\end{tabular}

Fuente: Elaboración propia

\footnotetext{
"Visión de Futuro" Año 18, Volumen N²5 N², Julio - Diciembre 2021 - Pág 81 - 99

URL de la Revista: http://visiondefuturo.fce.unam.edu.ar/index.php/visiondefuturo/index

URL del Documento: https://visiondefuturo.fce.unam.edu.ar/index.php/visiondefuturo/issue/view/21

ISSN 1668 - 8708 - Versión en Línea

E-mail: revistacientifica@fce.unam.edu.ar
} 


\section{5.- Análisis de los datos}

Las ponderaciones de los factores se procesaron en el software Matriz DAFO, reflejándose los resultados del análisis en la figura 2. Puede apreciarse que para la fase de pre inversión en Holguín se obtuvo un valor matricial de 34.5, encontrándose en el cuadrante II, zona de protección, debiéndose asumir estrategias de tipo defensivas que maximicen las fortalezas actuales y minimicen el efecto de las amenazas provenientes del entorno.

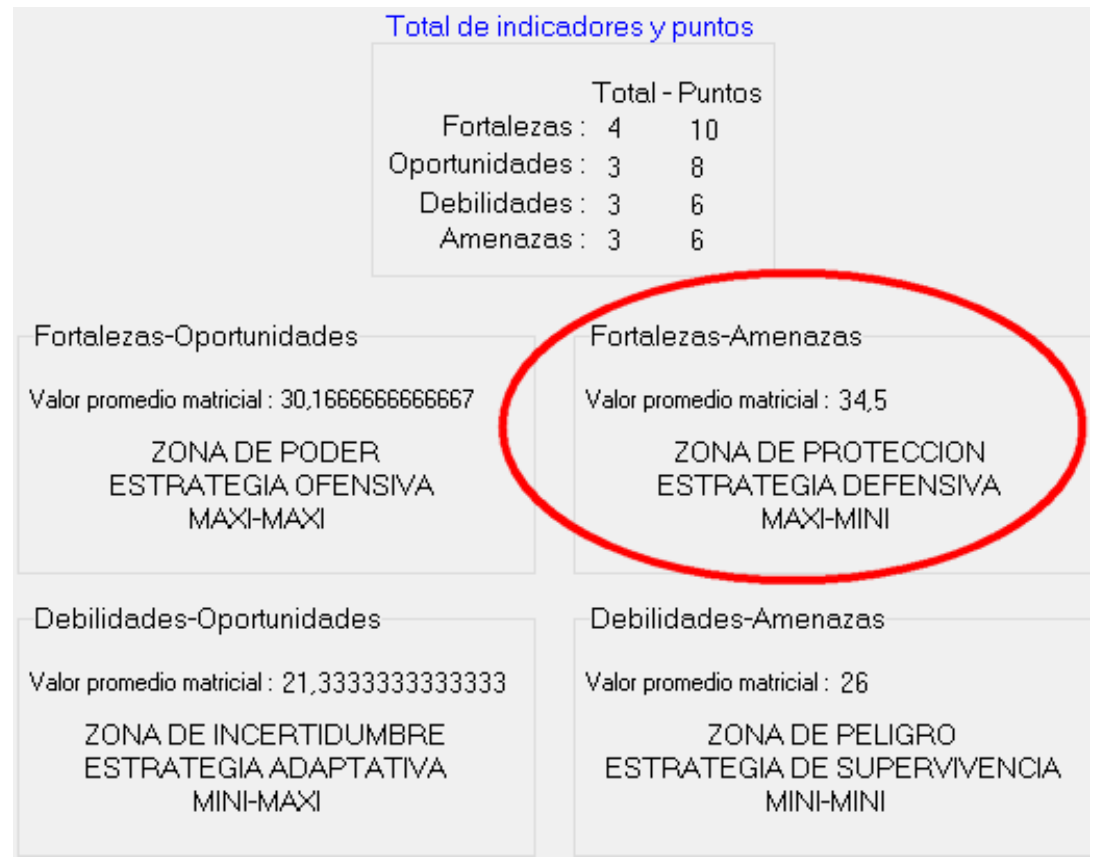

Figura N²: Resultados del análisis DAFO

Fuente: Software Matriz DAFO

En consecuencia, para potenciar las fortalezas actuales y contrarrestar el efecto de las amenazas identificadas se propone un diseño del subproceso pre inversión en Holguín, que contribuya a una mayor calidad y asertividad del mismo. Para esto se empleó la Casa de la Calidad o Despliegue de la Función Calidad (QFD), puesto que esta contempla la traducción de requerimientos externos al proceso/bien/servicio a características técnicas internas necesarias en la ejecución/producción del proceso/bien/servicio.

Constituye además un mecanismo formal para asegurar que las necesidades del cliente, expresadas en sus propios términos (la voz del cliente) sean traducidas al lenguaje técnico.

Para la aplicación de esta herramienta se desarrolló el esquema de trabajo siguiente:

1. Determinar los requerimientos del cliente (características reales).

2. Ponderar las características reales.

\footnotetext{
"Visión de Futuro" Año 18, Volumen No 25 N², Julio - Diciembre 2021 - Pág 81 - 99 URL de la Revista: http://visiondefuturo.fce.unam.edu.ar/index.php/visiondefuturo/index URL del Documento: https://visiondefuturo.fce.unam.edu.ar/index.php/visiondefuturo/issue/view/21 ISSN 1668 - 8708 - Versión en Línea 
3. Identificar las características sustitutas. Las características del proceso señaladas por el cliente (inversionistas, proyectistas, constructores) deben se transformadas en requisitos técnicos (requisitos de diseño equivalentes a las características sustitutas) que ejercen una influencia directa en las percepciones del cliente.

4. Desarrollar la matriz de relación central entre los requerimientos del cliente y las características de sustitutas. Esta indica el grado en que cada una de las características de sustitutas afecta las características señaladas por el cliente

5. Elaborar la Matriz de Relación Superior o Triangular (correlación entre las características sustitutas). Permite hacer cambios entre los pasos 3 y 4 con el fin de juzgar los posibles pros y contras entre las características sustitutas y las señaladas por el cliente.

6. Determinar la evaluación competitiva. Se obtiene el perfil competitivo del subproceso pre inversión en el contexto holguinero.

En conjunto con el grupo de expertos se realizó un levantamiento de los principales planteamientos de los diferentes organismos inmersos en procesos inversionistas en la provincia y que se desempeñan como inversionistas directos, proyectistas, constructores y demás sujetos del proceso inversionista (Consejo de Ministros, 2015), los que fueron tomados como características reales. Se aplicó y validó una segunda encuesta $(\alpha=0.81)$ al grupo de expertos para la ponderación de las características reales y su respectiva traducción al lenguaje técnico inherente al proceso inversionista y la fase de pre inversión en Holguín (tabla 3) así como, para clasificar la relación entre las características reales y sustitutas en: fuerte, media y débil reflejada en la figura 3.

Tabla N³: Traducción de las características reales

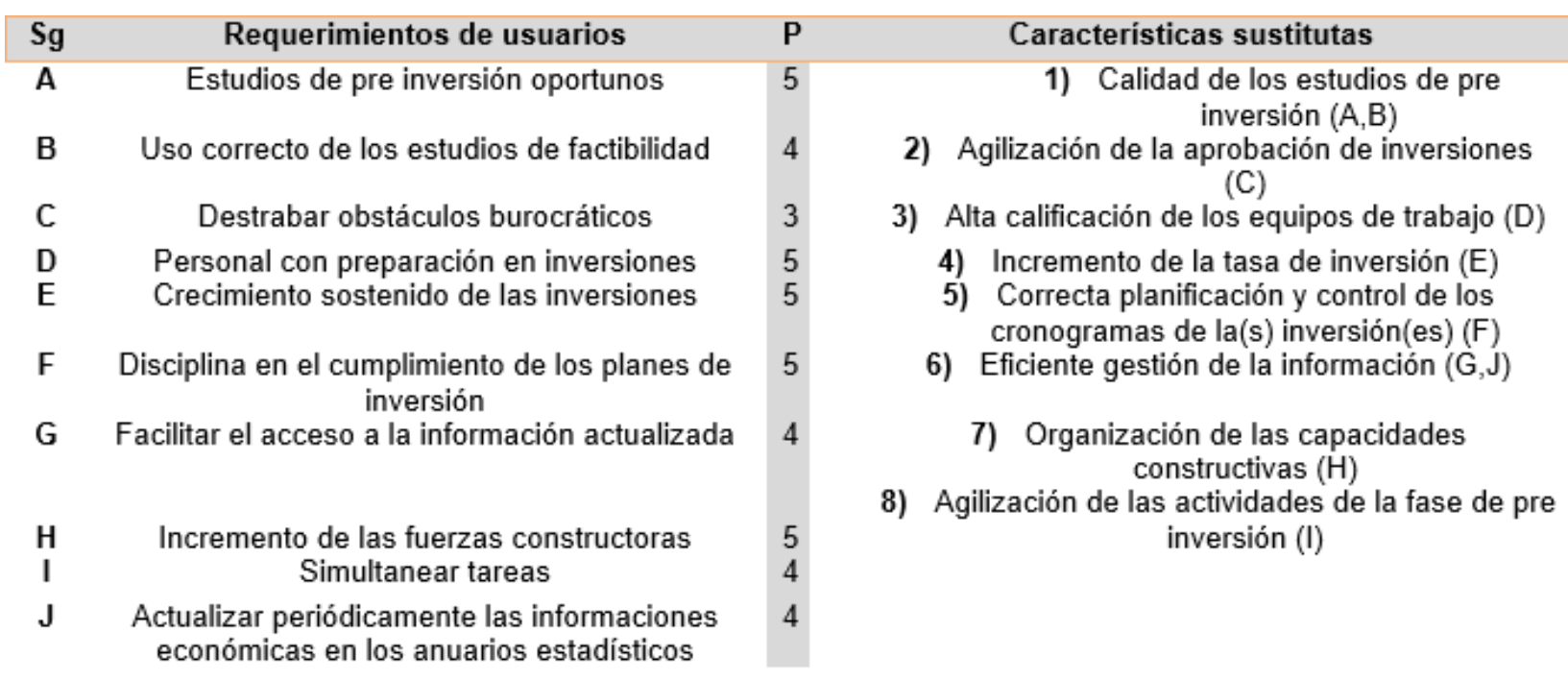

Fuente: Criterio de expertos

\footnotetext{
"Visión de Futuro" Año 18, Volumen No 25 N² 2, Julio - Diciembre 2021 - Pág 81 - 99

URL de la Revista: http://visiondefuturo.fce.unam.edu.ar/index.php/visiondefuturo/index

URL del Documento: https://visiondefuturo.fce.unam.edu.ar/index.php/visiondefuturo/issue/view/21

ISSN 1668 - 8708 - Versión en Línea

E-mail: revistacientifica@fce.unam.edu.ar
} 


\section{CUESTIONARIO}

Estimado colaborador tenemos a bien recoger sus consideraciones para ponderar las características reales de la fase de pre inversión del proceso inversionista e identificar la relación entre estas y las características técnicas o sustitutas.

Agradecemos su colaboración en la respuesta de este cuestionario y el esfuerzo en reportar los datos con alta confiabilidad. Los resultados obtenidos de su aplicación serán manejados con total confidencialidad ya que el estudio es estrictamente académico.

Instrucciones: lea cuidadosamente todo el documento, pondere las características reales acorde a la importancia que le concede dentro del proceso inversionista (1. Muy baja importancia 2. Baja importancia 3. Importancia media 4. Alta importancia 5. Importancia extrema)

Luego:

Califique la relación entre las características reales y sustitutas (1. Fuerte. 2. Media. 3. Débil)

\begin{tabular}{|c|c|c|c|c|c|c|c|}
\hline Característica real & Característica técnica & Relación & 1 & 2 & 3 & 4 & 5 \\
\hline Estudios de pre inversión fiables & \multirow{2}{*}{$\begin{array}{l}\text { Calidad de los estudios } \\
\text { de pre inversión }\end{array}$} & & & & & & \\
\hline $\begin{array}{l}\text { Correcta realización de los } \\
\text { estudios de factibilidad }\end{array}$ & & & & & & & \\
\hline $\begin{array}{c}\text { Destrabar obstáculos } \\
\text { burocráticos }\end{array}$ & $\begin{array}{l}\text { Agilización de la } \\
\text { aprobación de } \\
\text { inversiones }\end{array}$ & & & & & & \\
\hline $\begin{array}{c}\text { Personal con preparación en } \\
\text { inversiones }\end{array}$ & $\begin{array}{c}\text { Alta calificación de los } \\
\text { equipos de trabajo }\end{array}$ & & & & & & \\
\hline $\begin{array}{l}\text { Crecimiento sostenido de las } \\
\text { inversiones }\end{array}$ & $\begin{array}{l}\text { Incremento de la tasa } \\
\text { de inversión }\end{array}$ & & & & & & \\
\hline $\begin{array}{l}\text { Disciplina en el cumplimiento de } \\
\text { los planes de inversión }\end{array}$ & $\begin{array}{l}\text { Correcta planificación y } \\
\text { control de los } \\
\text { cronogramas de la(s) } \\
\text { inversión(es) }\end{array}$ & & & & & & \\
\hline $\begin{array}{l}\text { Facilitar el acceso a la } \\
\text { información actualizada }\end{array}$ & $\begin{array}{l}\text { Eficiente gestión de la } \\
\text { información }\end{array}$ & & & & & & \\
\hline $\begin{array}{l}\text { Incremento de las fuerzas } \\
\text { constructoras }\end{array}$ & $\begin{array}{c}\text { Organización de las } \\
\text { capacidades } \\
\text { constructivas }\end{array}$ & & & & & & \\
\hline Simultanear tareas & $\begin{array}{c}\text { Agilización de las } \\
\text { actividades de la fase } \\
\text { de pre inversión }\end{array}$ & & & & & & \\
\hline $\begin{array}{c}\text { Actualizar periódicamente las } \\
\text { informaciones económicas en } \\
\text { los anuarios estadísticos }\end{array}$ & $\begin{array}{l}\text { Eficiente gestión de la } \\
\text { información }\end{array}$ & & & & & & \\
\hline
\end{tabular}

Figura $N^{\circ} 3$ : Encuesta nivel de importancia y tipo de relación de las características reales y técnicas.

Fuente: Elaboración propia

Se realizó una selección de países de la región atendiendo al dinamismo en las inversiones y el crecimiento sostenido de estas, por cuanto, para el análisis de la competencia se tuvo en cuenta el desempeño de la fase de pre inversión dentro de los procesos inversionistas de Brasil, Colombia y Panamá (CEPAL, 2019). Para la evaluación competitiva se tuvo en cuenta el comportamiento de las características reales antes referidas en estos países y fueron calificados en una escala ascendente (1-5) por los expertos a partir del análisis del comportamiento de los criterios siguientes (CEPAL, 2019):

\footnotetext{
"Visión de Futuro" Año 18, Volumen No 25 N² 2, Julio - Diciembre 2021 - Pág 81 - 99 URL de la Revista: http://visiondefuturo.fce.unam.edu.ar/index.php/visiondefuturo/index URL del Documento: https://visiondefuturo.fce.unam.edu.ar/index.php/visiondefuturo/issue/view/21 ISSN 1668 - 8708 - Versión en Línea 
- Volumen de inversión total

- Crecimiento del PIB por concepto de inversiones

- Planes nacionales de desarrollo y su alcance

- Disposiciones legales, procedimientos y estándares de calidad referentes a la fase de pre inversión

Es preciso señalar que aunque Holguín representa una provincia, la evaluación competitiva contempló países atendiendo al grado de interconexiones existentes entre las provincias de estos países y sus respectivos Sistemas Nacionales de Inversión Pública.

\section{6.- Resultados}

Al desplegar la función de calidad se observó que las características técnicas (parte superior central figura 4) con mayor puntuación (parte inferior central figura 4) fueron la calidad de los estudios de pre inversión, la alta calificación de los equipos de trabajo, la correcta planificación y control de los cronogramas de la inversión y la eficiente gestión de la información. Por tanto, estas constituyen las características de calidad principales del subproceso de pre inversión.

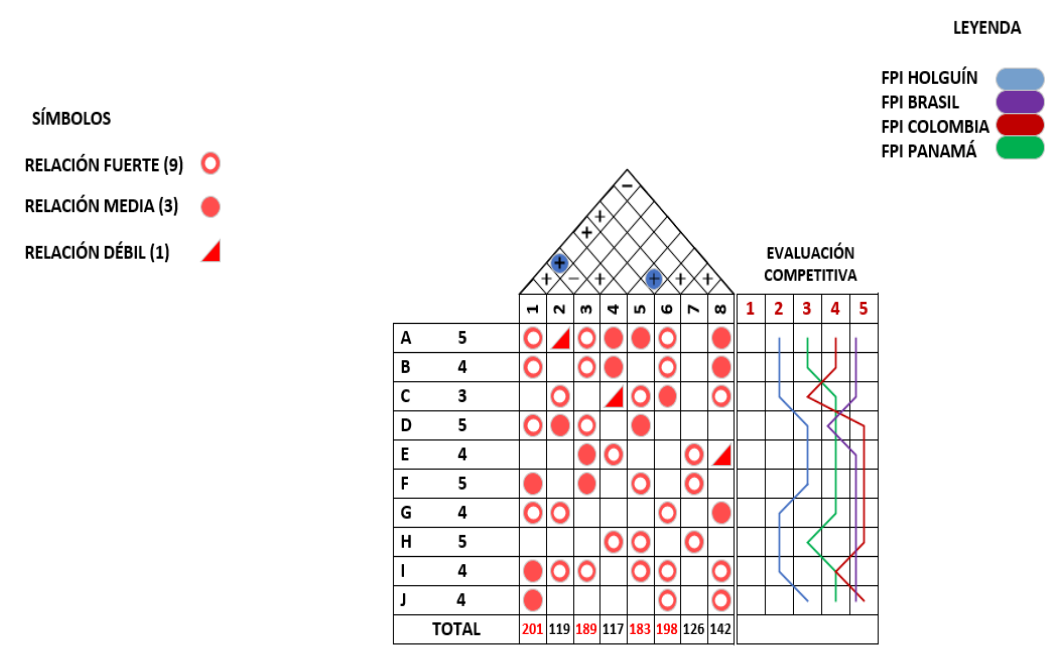

Figura $N^{\circ}$ 4: Casa de la Calidad aplicada a la fase de pre inversión en Holguín

Fuente: Elaboración propia

\footnotetext{
"Visión de Futuro" Año 18, Volumen N$^{0} 25$ No 2, Julio - Diciembre 2021 - Pág 81 - 99

URL de la Revista: http://visiondefuturo.fce.unam.edu.ar/index.php/visiondefuturo/index

URL del Documento: https://visiondefuturo.fce.unam.edu.ar/index.php/visiondefuturo/issue/view/21

ISSN 1668 - 8708 - Versión en Línea

E-mail: revistacientifica@fce.unam.edu.ar
} 
Se analizó el perfil competitivo de la fase de pre inversión en los procesos inversionistas de Brasil, Colombia y Panamá resultando el perfil de Brasil el más sólido en el área (parte lateral derecha Figura 4). Se realizó una comparación del perfil de Brasil con el de la provincia de Holguín obteniéndose los puntos más débiles de la fase de pre inversión. Las deficiencias en la realización de los estudios de pre inversión, los obstáculos burocráticos, el acceso a información actualizada y la imposibilidad de simultanear tareas representan los puntos más débiles de la FPI en Holguín.

Por otra parte, existen potencialidades de mejora en la preparación de los equipos de trabajo, el crecimiento sostenido de las inversiones y en el cumplimiento de los planes y cronogramas de las inversiones. Resulta preciso subrayar el vínculo de los puntos más débiles y las oportunidades de mejora con las características de calidad más importantes. Por tanto, contemplar estas últimas como requisitos para el diseño del subproceso pre inversión en Holguín constituye una vía objetiva de minimizar el efecto de las debilidades detectadas para un mejor aprovechamiento de las oportunidades identificadas en el perfil competitivo de la FPI en Holguín.

\section{7.- Propuesta de variables a considerar en el rediseño del proceso de pre inversión en Holguín}

La calidad de los estudios de pre inversión representa un factor indispensable para lograr el éxito de la inversión (Aguilera García et al., 2020). La correcta argumentación de la demanda y la depreciación en los estudios de mercado, la estimación oportuna del capital de trabajo y la realización de los estudios de pre y factibilidad técnico económico con la profundidad requerida coadyuvan al logro de la calidad y el éxito de esta fase y por ende del proceso en general. Por tanto, la evaluación de la calidad con que se desarrolla la fase de pre inversión se propone como parte del diseño del proceso siendo un elemento estratégico que aporta asertividad al mismo.

Por otra parte, la preparación y calificación de los equipos de trabajo inmersos en la ejecución de la FPI en Holguín constituye una condición necesaria para garantizar la asertividad de este subproceso (Sánchez et al., 2018). Por cuanto, deben intencionarse acciones de capacitación específicas para estos grupos de trabajo orientadas a aspectos técnicos, económicos y de calidad inherentes a los estudios y análisis que se realizan en el subproceso antes mencionado. Resulta coherente además, el enfoque de procesos en la gestión de la FPI teniendo en cuenta que su principal salida es la información a emplear en el resto de los subprocesos (ejecución-explotación y desactivación) que conforman el proceso

\footnotetext{
"Visión de Futuro" Año 18, Volumen N²5 N², Julio - Diciembre 2021 - Pág 81 - 99

URL de la Revista: http://visiondefuturo.fce.unam.edu.ar/index.php/visiondefuturo/index

URL del Documento: https://visiondefuturo.fce.unam.edu.ar/index.php/visiondefuturo/issue/view/21

ISSN 1668 - 8708 - Versión en Línea

E-mail: revistacientifica@fce.unam.edu.ar
} 
inversionista.

Otro elemento importante a considerar en el diseño del subproceso pre inversión en el contexto holguinero es el cumplimiento de los plazos o cronogramas, aspecto que posee un peso específico significativo en el desarrollo exitoso de la fase de pre inversión y del proceso inversionista en general (Garro, 2015). Su papel en el control del desarrollo de la inversión de forma integral influye en la eficiencia del subproceso (Mahlotra, 2004; López y García, 2012; Mesa, 2012; Valarezo et al., 2016; Martín et al., 2017; Almarales et al., 2019).

El cumplimiento estricto de los cronogramas debe estar respaldado por una efectiva simultaneidad de tareas en cada una de las actividades del subproceso pre inversión. La simultaneidad de tareas si bien no representa una vía esquemática para dinamizar y asegurar el cumplimiento de los plazos, debe respaldarse legalmente como principio vertebral del subproceso teniendo en cuenta la especificidad de cada proyecto de inversión y sus características.

Por tanto, generalizar el empleo de herramientas de temporización y planificación como el Enfoque Marco-Lógico (Sánchez, 2007; Sainz et al., 2015; Monroy, 2018), método CPM (Lao, 2013) y el diagrama de Gantt (Honores, 2017), constituye un punto de inflexión para el logro de una mejor planificación, ejecución y control de los cronogramas desde el subproceso pre inversión.

Sin embargo, los elementos antes expuestos deben estar sustentados en una óptima gestión de la información que transversalice todas las actividades del subproceso pre inversión. La actualización de los principales anuarios estadísticos y la accesibilidad a datos reales del mercado constituyen medidas emergentes para lograr una mayor fiabilidad en los análisis y pronósticos realizados en la fase de pre inversión.

\section{CONCLUSIÓN}

El subproceso pre inversión en Holguín se ubica en la cuadrante de protección, debiéndose adoptar estrategias defensivas orientadas a potenciar las fortalezas para atenuar las amenazas externas.

Las características de calidad más representativas en el diseño del subproceso pre inversión en Holguín son: la calidad de los estudios de pre inversión, la alta calificación de los equipos de trabajo inmersos en el subproceso, la correcta planificación y control de los cronogramas de la inversión y la eficiente gestión de la información.

El subproceso de pre inversión en Holguín presenta como puntos débiles dentro de su perfil competitivo las deficiencias en la realización oportuna de los estudios de pre inversión, los obstáculos en el acceso a información actualizada para la realización de los estudios de

\footnotetext{
"Visión de Futuro" Año 18, Volumen No 25 N² 2, Julio - Diciembre 2021 - Pág 81 - 99

URL de la Revista: http://visiondefuturo.fce.unam.edu.ar/index.php/visiondefuturo/index

URL del Documento: https://visiondefuturo.fce.unam.edu.ar/index.php/visiondefuturo/issue/view/21

ISSN 1668 - 8708 - Versión en Línea

E-mail: revistacientifica@fce.unam.edu.ar
} 
pre inversión y las brechas en el la realización simultánea de tareas que favorezcan la agilidad en la ejecución de la fase de pre inversión.

Las oportunidades de mejora del subproceso de pre inversión en el contexto holguinero, acorde al perfil competitivo del mismo, están asociadas a una mejor preparación de los equipos de trabajo, el crecimiento sostenido de las inversiones y el cumplimiento de los planes y cronogramas de la inversión.

Los puntos débiles y las oportunidades de mejora del subproceso pre inversión en Holguín están vinculados con las características de calidad más significativas y representan requisitos para el diseño del subproceso.

El diseño propuesto del subproceso de pre inversión en el contexto holguinero considera las características de calidad más valoradas por los clientes, los puntos débiles y las oportunidades de mejora, por cuanto contribuye a la calidad y asertividad de la fase de pre inversión en Holguín.

\section{REFERENCIAS}

Aguilera, L. A. et al., "Dimensiones y variables de la calidad de la fase de pre inversión". Ciencias Holguín, Enero-Marzo 2020, Volumen:26, №1, p.15, ISSN: 1027-2127, http://www.ciencias.holguin.cu

Almarales, L. M. et al., "La tasa de descuento en la gestión empresarial del proceso inversionista cubano". Ciencias Holguín, Abril-Junio 2019, Volumen 25, №2, pgs.15-29, ISSN: 1027-2127, http://www.redalyc.org/articulo.oa?id=181559111002

BOLIVIA, Reglamento básico de preinversión de Bolivia. La Paz, 115 C.F.R. (2015). http://www.rubenapaza.com/2015/11/reglamento-basico-de-preinversion-de.html [consultada el 19/05/20]

Cabrera, V. M. y Paredes, D. M. (2016). Modelo de evaluación de sostenibilidad para el Sistema Nacional de Inversión Pública del Perú. Tesis de Licenciatura en Gestión Pública. $\begin{array}{llllll}\text { Pontificia Universidad } & \text { Católica } & \text { del } & \text { Perú, } & \text { p } & 135 .\end{array}$ http://tesis.pucp.edu.pe/repositorio/handle/20.500.12404/12527

COLOMBIA, Cámara Colombiana de la Infraestructura, http://www.infraestructura.org.co/bibliotecas/VPT/PREINVERSION\%20EN\%20PROYECTOS \%20DE\%20INFRAESTRUCTURA\%20(JULIO\%202016).pdf, Bogotá (2016). Preinversión en proyectos de infraestructura. Consultado: 5/09/2019, 2019.

Carabias, S. (2002). Análisis Estocástico de los Procesos de Inversión. Tesis de Maestría. Universidad Complutense de Madrid, Madrid, p-96, http://eprints.ucm.es/3640

\footnotetext{
"Visión de Futuro" Año 18, Volumen N²5 N² 2, Julio - Diciembre 2021 - Pág 81 - 99

URL de la Revista: http://visiondefuturo.fce.unam.edu.ar/index.php/visiondefuturo/index

URL del Documento: https://visiondefuturo.fce.unam.edu.ar/index.php/visiondefuturo/issue/view/21

ISSN 1668 - 8708 - Versión en Línea

E-mail: revistacientifica@fce.unam.edu.ar
} 
Comisión Económica Para América Latina [CEPAL] (2019). Reportes de las perspectivas económicas de América Latina 2019: Desarrollo en transición, Economía, Transformación, Editorial OCDE/NACIONES UNIDAS/CAF/UE (Ed.), (OA-03-19-518-ES-N ed., pgs. 252). http://doi.org/10.1787/g2g9ff1a-es.

Consejo de ministros, "Decreto Ley 327. Reglamento del proceso inversionista". Gaceta Oficial de la República de Cuba, Volumen IV, Junio-Diciembre 2015, № 5, pgs:27-59, ISSN: 1682-7511, http://www.dncontabilidad.sld.cu/Doc/GO\%20327_X_5_2015.pdf

Duffus, D. et al., "Estudio técnico para la evaluación de proyectos en la fase de pre inversión en Cuba". OBSERVATORIO DE LA ECONOMÍA LATINOAMERICANA, diciembre 2018, ISSN: 1696-8352, http://www.eumed.net/rev/oel/2018/05/evaluacion-proyectoscuba.html

Elizondo, R. "QFD-Herramienta de calidad base para una estrategia de comunicación. Caso aplicado en un micro beneficio costarricense". FISEC-Estrategias, Volumen 1, 2019, №1, pgs.181-201, ISSN:

1669-4015, http://redmarka.net/ra/usr/9/1247/fisec_estrategias_n15v3pp181_201.pdf

Foschiatti, A. M. y Alberto, J. A. "El uso de matrices DAFO como herramientas de gestión y análisis geográfico". GEOGRAFICA DIGITAL,Volumen 9, enero 2019, №18, pgs. 1-11, ISSN: 1668-5180, http://revistas.unne.edu.ar/index.php/geo/article/view/2235

Garro, E. R. (2015). Evaluación de los estudios de pre inversión declarados viables a nivel de perfil del proyecto en la municipalidad distrital de San Marcos. Tesis de diploma, Universidad Nacional Santiago Antúnez De Mayolo, Huaraz, Perú. http://repositorio.unasam.edu.pe/handle/UNASAM/1188

Honores, D. A. (2017). Utilización de diagramas de Gantt para la programación y control de un proyecto en la empresa "DH". Tesis de grado. Universidad Técnica de Machala, Machala. http://repositorio.utmachala.edu.ec/handle/48000/10013

Kim, K. J. et al. "Robust QFD: framework and a case study. Quality and reliability engenieering international", Volumen 23, febrero 2007, № 1, pgs. 31-44, ISSN: 0748-8017, http://onlinelibrary.wiley.com/doi/abs/10.1002/qre.821

Lao, Y. O. (2013). Procedimiento para el perfeccionamiento de las funciones de la administración de operaciones en la EMPA Holguín. Tesis de maestría en Ingeniería Industrial, Universidad de Holguín, Cuba. http://dx.doi.org/10.13140/RG.2.2.16412.39046.

López, L.V. y García, V. "El proceso inversionista y la financiación de inversiones en Cuba: deficiencias, limitaciones y retos". Economía y Desarrollo, Volumen 148, enero-marzo 2012, №2, pgs. 124-138, ISSN: 2518-0983,

http://www.econdesarrollo.uh.cu/index.php/RED/article/view/271

\footnotetext{
“Visión de Futuro" Año 18, Volumen No $^{\circ} 5$ Nº 2, Julio - Diciembre 2021 - Pág 81 - 99

URL de la Revista: http://visiondefuturo.fce.unam.edu.ar/index.php/visiondefuturo/index

URL del Documento: https://visiondefuturo.fce.unam.edu.ar/index.php/visiondefuturo/issue/view/21

ISSN 1668 - 8708 - Versión en Línea

E-mail: revistacientifica@fce.unam.edu.ar
} 
Mahlotra, N. (2004). Investigación de mercados. Un enfoque aplicado,Editorial Quintanar Duarte, Enrique Ed. 4ta ed. Vol. 4th. México: Pearson Prentice-Hall., ISBN: 970-2604915.

http://books.google.es/books?hl=es\&lr=\&id=SLmEbIVK2OQC\&oi=fnd\&pg=PP19\&dq=Investi gaci\%C3\%B3n+de+mercados.+Un+enfoque+aplicado+de+malhotra\&ots=wbdh36Yhm2\&sig $=$ AzPItXGpFbPTyuhpHkVPsyJ4i8\#v=onepage \&q=Investigaci\%C3\%B3n\%20de\%20mercado s.\%20Un\%20enfoque\%20aplicado\%20de\%20malhotra\&f=false

Martín, A. M. et al. "Análisis crítico de la inversión en energías renovables. Enfoque socioeconómico". Cofín Habana, Volumen 11, enero-marzo 2017, № 2, pgs. 69-90, ISSN: 2073-6061,http://scielo.sld.cu/scielo.php?script=sci_arttext\&pid=S2073-

$60612017000200006 \& n r m=$ iso

Mesa, G.M. (2012). La evaluación financiera y social de proyectos de inversión, Editorial Félix Varela (La Ed. 3th ed.). La Habana, Cuba: Facultad de Economía Universidad de La Habana, ISBN: 959-16-0424-6, pg 89, http://catalogo.bnjm.cu/cgi-bin/koha/opacdetail.pl?biblionumber $=112267$

Monroy, G. A. (2018). Propuesta para la implementación de la matriz del marco lógico en la planificación, administración y ejecución de proyectos de construcción. Tesis de grado, Universidad de San Carlos de Guatemala, Guatemala. http://www.repositorio.usac.edu.gt/9996

Ochoa, Y. (2019). Contribución a la evaluación de la calidad de la fase de pre inversión. Tesis de grado en Ingeniería Industrial), Universidad de Holguín, Holguìn. http://repositorio.uho.edu.cu/jspui/handle/uho/166

Olivera, A. P. et al. "El análisis DAFO y los objetivos estratégicos. La matriz DAFO en la gestión estratégica", Volumen 1, diciembre 2012, №6, ISSN: 1696-8360, http://www.eumed.net/ce/2011a/domh.zip

Paño, E. (2016). Desfases en la gestion de los proyectos de inversión pública en la etapa de preinversion. Tesis de maestría, Universidad Nacional de San Antonio Abad del Cusco, Perú. http://repositorio.unsaac.edu.pe/handle/UNSAAC/2957

Peña, I. G. et al. "Estudio de factibilidad para la implementación de un ciclo orgánico de rankine en pozos de extracción de petróleo". Revista EIA, Volumen 23, 2015, pgs. 137-148, ISSN: 1794-1237, DOI: http://dx.doi.org/10.24050/reia.v0i0.533,

Pérez, M (2011). Estudio de factibilidad económica de las mesas rebatibles para la construcción de viviendas en la Empresa Constructora Militar \#4. Tesis de maestria, Universidad de Matanzas "Camilo Cienfuegos". Matanzas.

\footnotetext{
"Visión de Futuro" Año 18, Volumen No 25 N², Julio - Diciembre 2021 - Pág 81 - 99

URL de la Revista: http://visiondefuturo.fce.unam.edu.ar/index.php/visiondefuturo/index

URL del Documento: https://visiondefuturo.fce.unam.edu.ar/index.php/visiondefuturo/issue/view/21

ISSN 1668 - 8708 - Versión en Línea

E-mail: revistacientifica@fce.unam.edu.ar
} 
Reich, Y. y Levy, E. "Managing product design quality under resource constraints". International Journal of Production Research, Volumen 42, 2004, №13, 2560-2572, ISSN: 0020-7543, DOI: http://doi.org/10.1080/0020754042000208394,

Rodríguez, A. (2019). Procedimiento para la evaluación de la calidad de la fase de pre inversión. Tesis de grado en Ingeniero Industrial, Universidad de Holguín, Holguín. http://repositorio.uho.edu.cu/jspui/bitstream/uho/5936/1/Anisleidis\%20Rodr\%C3\%ADguez\%2 OAlmaguer.pdf

Sáenz, T. (2013). Apuntes para una mejor interpretación de los estudios de factibilidad.http://centroazucar.uclv.edu.cu/media/articulos/PDF/2015/4/A_NDICE\%20ACUM ULATIVO\%202015.pdf [consultada el 15/03/19].

Sainz, R. O. et al. "Determinación de los objetivos parciales de un proyecto, aplicando la ruta más larga". UPGTO Management review,Volumen 1, 2015, № 2, pgs.18-43, ISSN: 2007-977X, http://dialnet.unirioja.es/servlet/articulo?codigo=5822193

Sánchez, I. R. y Ledesma, Z. M. "Proceso inversionista eficiente: Papel del estudio de preinversión en las condiciones de Cuba". EKOTEMAS, Volumen 1, 2015a, №1, pgs. 1-11, ISSN: 2414-4681, http://www.ekotemas.cu/index.php/ekotemas/article/view/94

Sánchez, I. R. y Ledesma, Z. M. "Gestión integral del proceso inversionista cubano: Evaluación del impacto de la capacitación en Villa Clara". Cofín Habana, Volumen 12, 2018, №2, $\quad$ pgs. 366-380, ISSN: 2073-6061, http://scielo.sld.cu/scielo.php?script=sci_arttext\&pid=S2073-60612018000200026

Sánchez, N. "El marco lógico. Metodología para la planificación, seguimiento y evaluación de proyectos". Visión Gerencial, Volumen 1, 2007, №2, pgs. 328-343, ISSN: 13178822, http://www.redalyc.org/articulo.oa?id=465545876012

Sapag, N. (2011). Proyectos de inversión. Formulación y evaluación. Editor Maluf, Inés Fernández Ed. 2nd ed. Chile, Editorial Pearson Educación de Chile S.A, ISBN: 978-956-343106-3. http://www.delfabro.cl/preparacinyevaluacindeproyectos_nassirsapag5edi.pdf

Sardiñas, G. R. (2016). Gerencia del proceso inversionista para introducir un proyecto de una mejor fábrica de azúcar en Antonio Sánchez. Tesis de Maestría, Universidad Central de Las Villas Martha Abreu. http://dspace.uclv.edu.cu:8089/handle/123456789/7522

Sarmiento, L. I. et al. "Aplicación de una Metodología de Mejora de Procesos basada en el Enfoque de Gestión por Procesos, en los Modelos de Excelencia y el QFD en una empresa del sector de confecciones de Barranquill". Ingeniería y Desarrollo, Volumen 1, 2004, №16, pgs. 45-58, ISSN: 0122-3461, http://www.rdalyc.org/articulo.oa85216004

\footnotetext{
"Visión de Futuro" Año 18, Volumen N²5 N² 2, Julio - Diciembre 2021 - Pág 81 - 99

URL de la Revista: http://visiondefuturo.fce.unam.edu.ar/index.php/visiondefuturo/index

URL del Documento: https://visiondefuturo.fce.unam.edu.ar/index.php/visiondefuturo/issue/view/21

ISSN 1668 - 8708 - Versión en Línea

E-mail: revistacientifica@fce.unam.edu.ar
} 
Guatemala, Secretaría de Planificación y Programación de la Presidencia en Guatemala. (2002). "Ciclo de vida de un proyecto de inversión". Disponible en: http://snip.segeplan.gob.gt/sinip/html/portal/temp/mnl_ciclo.htm, Consultado: 5/09/2019, 2019

Shen, $X$. et al. "Listening to the future voice of the customer using fuzzy trend analysis in QFD". Quality Engineering, Volumen 13, abril-junio 2001, №3, pgs. 419-425, ISSN: 08982112, DOI: http://doi.org/10.1080/08982110108918670,

Thompson, M. (2009). Etapas de la fase de preinversión. Retrieved from http://todosobreproyectos.blogspot.com

Valarezo B. et al. "Estudio de factibilidad en el sistema de dirección por proyectos de inversión". Ingeniería Industrial, Volumen 37, 2016, №3, pgs. 305-312, ISSN: 1815-5936, http://scielo.sld.cu/scielo.php/pid=S18155936201600030009\&script=sci_arttext\&tlng=pt

Vera, L. H. (2015). Factores que contribuyeron a prolongar la duración del proceso de formulación de los estudios de pre inversión (perfil, pre - factibilidad y factibilidad) del proyecto de inversión pública denominado "mejoramiento de la atención de las personas con discapacidad de alta complejidad en el instituto nacional de rehabilitación. Tesis de maestría, Pontificia Universidad Católica del Perú, Lima. http://tesis.pucp.edu.pe/repositorio/handle/20.500.12404/5908

Villafuerte, R. E. (2016). Lineamientos para mejorar la gestión de proyectos de construcción de los gobiernos regionales y locales en la etapa de preinversión, bajo el enfoque de "lean construction". Tesis de maestría, Pontificia Universidad Católica del Perú, Lima, Perú. http://tesis.pucp.edu.pe/repositorio/handle/20.500.12404/8645

Wang, L. et al. "Selection criteria and feasibility of the inversion model for azimuthal electromagnetic logging while drilling (LWD)". Petroleum Exploration and Development, Volumen 45, diciembre 2018, №5, pgs. 974-982, ISSN: 1876-3804, DOI: http://doi.org/10.1016/S1876-3804(18)30101-0

\section{RESUMEN BIBLIOGRÁFICO}

\section{Luis Alexis Aguilera García}

Ingeniero Industrial por la Universidad de Holguín (2015). Profesor Asistente. Metodólogo del departamento de Comercialización de la Universidad de Holguín. Sus intereses investigativos versan sobre la Gestión de la calidad en el proceso inversionista.

\section{Yosvani Orlando Lao León}

Doctor en Ciencias Técnicas por la Universidad de Holguín (2017). Profesor Auxiliar del Departamento de Turismo de la Faculta de Ciencias Empresariales y Administración. Sus intereses investigativos versan sobre la Investigación de Operaciones y la Inteligencia Artificial aplicadas a la Cadena de "Visión de Futuro" Año 18, Volumen N²5 N², Julio - Diciembre 2021 - Pág 81 - 99 URL de la Revista: http://visiondefuturo.fce.unam.edu.ar/index.php/visiondefuturo/index URL del Documento: https://visiondefuturo.fce.unam.edu.ar/index.php/visiondefuturo/issue/view/21 ISSN 1668 - 8708 - Versión en Línea E-mail: revistacientifica@fce.unam.edu.ar 
Suministros y el proceso inversionista.

\section{Maira Rosario Moreno Pino}

Doctora en Ciencias Pedagógicas por la Universidad de Oriente, Santiago de Cuba (2003). Profesora Titular. Jefa de la disciplina Calidad en la carrera de Ingeniería Industrial en la Universidad de Holguín. Sus líneas de investigación fundamentales son la Gestión de la Calidad y el proceso inversionista.

\section{Yumelys Lores Rodríguez}

Ingeniera Industrial por la Universidad de Holguín (2010). Profesora Instructora. Especialista comercial e inversiones en la empresa SEISA Holguín. Sus intereses investigativos versan sobre la evaluación y mejora del proceso inversionista.

\footnotetext{
"Visión de Futuro" Año 18, Volumen No 25 No 2, Julio - Diciembre 2021 - Pág 81 - 99 URL de la Revista: http://visiondefuturo.fce.unam.edu.ar/index.php/visiondefuturo/index URL del Documento: https://visiondefuturo.fce.unam.edu.ar/index.php/visiondefuturo/issue/view/21 ISSN 1668 - 8708 - Versión en Línea E-mail: revistacientifica@fce.unam.edu.ar
} 